\title{
Design and Evaluation of Interaction Techniques Dedicated to Integrate Encountered-Type Haptic Displays in Virtual Environments
}

\author{
Víctor Mercado \\ Univ Rennes, INSA, Inria, CNRS, IRISA
}

\author{
Maud Marchal \\ Univ Rennes, INSA, Inria, CNRS, IRISA
}

\author{
Anatole Lécuyer* \\ Univ Rennes, Inria, CNRS, IRISA
}

\begin{abstract}
Encountered-Type Haptic Displays (ETHDs) represent a field of haptic displays with the premise of not using any type of actuator directly in contact with the user skin, thus providing an alternative integration of haptic displays in virtual environments. In this paper, we present novel interaction techniques (ITs) dedicated to ETHDs. The techniques aim at addressing the issues commonly presented for these devices such as limited contact areas, lags and unexpected collisions with the user. First, our paper proposes a design framework based on several parameters defining the interactive process between user and ETHD (input, movement control, displacement and contact). Five techniques based on different ramifications of the design space framework were conceived, respectively named: Swipe, Drag, Clutch, Bubble and Follow. Then, a use-case scenario was designed to depict the usage of these techniques on the task of touching and coloring a wide, flat surface. Finally, a user study based on the coloring task was conducted to assess the performance and user experience for each IT. Results were in favor of Drag and Clutch techniques which are based on manual surface displacement, absolute position selection and intermittent contact interaction. Taken together our results and design methodology pave the way to the design of future ITs for ETHDs in virtual environments.
\end{abstract}

Index Terms: Encountered-Type Haptic Displays-Interaction Techniques-Haptic Rendering - Human-Machine Interaction;

\section{INTRODUCTION}

Virtual Reality (VR) can be enriched by haptic technologies to integrate the sense of touch in virtual environments (VEs). Having tactile feedback when interacting with virtual environments leads to a stronger immersion and the possibility of having more sophisticated interactions between user and system [7]. Among the various haptic technologies, Encountered-Type Haptic Displays (ETHDs) provide an opportunity for exploiting the potential of a more natural integration between haptic displays and VEs. ETHDs represent a field of haptic displays with the premise of not using any type of actuator directly in contact with the user skin, therefore only providing stimulation when it is needed [26,31]. To achieve this type of feedback, ETHDs rely on a tracking system that permits to know the user's location and thus positioning themselves to "encounter" the user at any required point during the interaction. When interacting with VEs, Head-Mounted Displays (HMDs) are generally used in ETHD systems to hide the haptic device and to contextualize the task to be carried out by the user [36].

ETHDs aim to reduce the constraints of wearable haptic displays by physically separating users from the haptic device and by providing contact only when the user desires to [26,31]. ETHDs can either rely on end-effectors that contain the haptic information to be perceived $[4,29,36]$ or by acting as proxies [3]. This permits a handfree interaction similar to reality. Nevertheless, ETHDs' dependency

*e-mail: victor.mercado, maud.marchal, anatole.lecuyer@inria.fr on external actuators that are not in constant contact with the user brings aspects to be considered in order to provide convincing haptic feedback. For instance, the dexterity of ETHDs for repositioning themselves with the appropriate speed, accuracy and safety measures should be taken into account $[19,33]$. The task of displacing the robot over different positions to render a large volume of haptic feedback often leads researchers to constrain the contact area and thus limiting the haptic feedback to a small area [34]. The elapsed time for calculating an ideal encounter position can produce lag and compromises both users' immersion and surface perception. Additionally, this lag often leads to unexpected user-device collisions or leads the user to touch the void instead of encountering the surface to be touched.

In this paper, we propose to introduce novel interaction techniques (ITs) dedicated to ETHDs to address current field issues. We designed a set of five ITs under a proposed design space framework. The novelty of our approach relies on the conceptualization and inclusion of a design space that comprehends different possibilities to conceive ITs for ETHDs. In addition, the designed ITs represent a novel approach in the interaction process in the aforementioned haptic displays. The global setup of our ETHD system is shown in Fig. 1. The techniques were mainly designed to enable the user to interact with virtual, flat, and wide surfaces using one finger. We chose to illustrate our techniques through a use-case scenario with a shape coloring task. This task was chosen since it requires to interact with a large surface where contact information is crucial under a large surface exploration. We chose to assess the ITs performance through a user study with a similar shape coloring task. To evaluate the techniques, both quantitative measurements and subjective questionnaires were used in order to identify the features that could be taken into account for designing an optimal interaction technique dedicated to ETHDs.

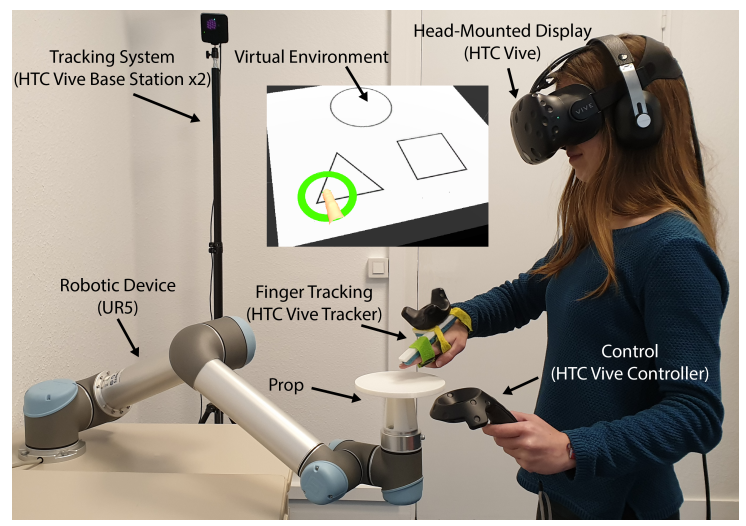

Figure 1: Global setup of our system. The grounded ETHD system (bottom-left) renders a large flat surface by positioning a physical prop (bottom-center) under the user's finger using different ITs. The task proposed in our use-case scenario consists in shape coloring in a VE (top-center). The user tracking system comprises the HTC Vive Base Stations (top-left), the Vive tracker and controller (middle-center) and the Vive HMD (top-left). 
The contributions of the paper are the following:

- A design space for interaction techniques dedicated to ETHDs.

- A set of 5 interaction techniques designed for optimizing the haptic rendering capacity of ETHDs in a scenario involving the exploration of a wide and flat surface.

- A use-case scenario to contextualize the application of the conceived techniques for haptic exploration in the task of shape coloring.

- A user study for assessing the performance of the proposed interaction techniques, both with quantitative and subjective measurements.

The paper is structured as follows: related work in ETHD interaction strategies is presented in Section 2. The proposed ITs are introduced and explained in Section 3. The use-case scenario for contextualizing our approach is showcased in Section 4. Section 5 presents the user study carried out to evaluate the formerly introduced techniques. Results and insights from the user study are discussed in Section 6. Finally, general remarks and conclusions are commented in in Section 7.

\section{Related Work}

ETHDs depend on either passive or active end-effectors as the main tool for rendering haptic feedback. The end-effector can be used on its entirety for having a complete object-surface representation in the VE or a partial surface representation that will depend on more sophisticated interaction strategies for rendering a surface larger than the end-effector. This section introduces the two main interaction strategies that have been considered by researchers to cope with constraints coming from both actuators and haptic end-effectors. The first paragraph covers the strategies considered for ETHDs that possess an end-effector with shape properties that coincide through several features with their virtual counterparts. These strategies mainly aim to have an accurate object-surface representation in both virtual and real environments. We will refer to static contact area for this category since the contact area does not have any motion during the user interaction. The second paragraph discusses related work on ETHD interaction strategies where the end-effector has a limited size compared to the virtual shape to be touched in the VE. To deal with this limited size, these strategies generally employ visuo-haptic illusions or users' attention redirection. We will refer to moveable contact area for this category.

\subsection{Static Contact Area}

Providing a static contact area for interaction implies to have a defined surface to touch per object in a VE assisted by an ETHD. Research has considered the possibility of providing a completeobject representation using end-effectors with the exactly similar shape properties than the objects to be touched in the VE. Having a complete object-surface representation signifies that the contact area represents the entirety of the object to be touched. This permits the users to perform more complex haptic exploratory procedures since the haptic display acts as the provider of the entire object to be touched. The advantage of this type of representations is that it is possible to provide a complete object-surface mapping. Yet, the constraints of the actual size of the end-effector can become cumbersome [1, 6, 15, 18,34,35]. Unmanned air vehicles have also been used in the field by profiting from the drone's properties to use the device as a proxy permitting haptic exploratory procedures related to enclosure and contour following. In the work of Abtahi et. al. [3], the contact area in the task of shoe-box displacing was inferred due to the volume mapping between the physical configuration of a net covering the drone and the shape of the virtual box.

Nevertheless, when the end-effector can only cover a part of the surface to be touched, the system strongly relies on visual metaphors that suggest the area that can actually be touched. In the work of Araujo et. al. [4], virtual shoes are used as the visual metaphor for indicating the user where to touch as a mean to perceive the shoe texture. In this case, the widest area to touch is the toe cap and thus the device's end-effector is placed there for providing the haptic feedback. In the work of Kim et. al. [19], a virtual door is centered in front of the user. The users were asked to push the door. This door-pushing metaphor permitted the users to push the door directly located in front of them without explicitly signaling the contact area.

Some ETHD systems have also considered to display the contact area in the VE. These depictions range from highlighting either the whole volume that can be touched $[8,10]$ or by highlighting a contact area represented in a virtual surface [3,29]. Users can choose the patch location depending on the system capabilities of positioning [3, 20,21,29].

\subsection{Moveable Contact Area}

Several ETHDs address the challenge of rendering surfaces larger than the physical device being held by this type of haptic displays. In order to explore a surface larger than the end-effector, the community has been looking forward to displace the contact area. An adequate integration between interaction strategy and end-effector's physical properties permits to recreate different surfaces that can go beyond what is physically represented on the device [4, 8, 13, 24, 27, 33]. Previous works have relied on strategies such as visuo-haptic illusion, user redirection and active surface displacement.

Visuo-haptic illusions have been used in ETHDs systems as a mean to give the sensation of touching large surfaces. The work of Abtahi \& Follmer [2] explores this type of illusions providing haptic feedback with a pin-based ETHD. The authors managed to increase the resolution of their system by adjusting the scale of the elements to be touched both in the real (the rendered shape by the pin display) and in the virtual environments. This work additionally redirected the users' contact with the shape display to increase and optimize the rendering space. User redirection has been considered for more complex ETHD systems such as integrating an omni-directional treadmill to redirect the user's location to a reachable area for the display [33]. The work of Whitmire et. al. [34] profits from a virtual poker table to be touched as the metaphor and the integration of a spinning surface below the user's finger for rendering different textures when users explore the virtual surface.

In order to provide natural haptic feedback for most of the elements of a VE, ETHDs using a moveable contact area represent a promising technological alternative for haptic display. They allow a more natural integration of haptic technologies within VEs. They also propose the possibility to explore a larger surface area by moving adequately during the interaction. However, ETHDs using a moveable contact area have also some technological limitations that can break the contact information due to latency during repositioning. Therefore, in this paper, we focus on the design of ITs that address these limitations and allow the interaction with a flat and wide surface area.

\section{INTERAction TEChNiques DESIGN}

This paper proposes a design space to explore and study different ITs for ETHDs. A set of 5 techniques were conceived based on several possibilities depicted in the design space. The first subsection discusses the design space as well as the features considered to cope with ETHDs' limitations. The second subsection explains the conceived techniques and their features. Fig. 3 provides a visual description for all the proposed techniques.

\subsection{Design Space}

To provide the sensation of touching a wide and flat surface area, we studied how to design ITs for ETHDs that could integrate the process of rendering a tangible surface without affecting users' immersion in the VE. We propose an IT design space based on previous work done in the domains of ETHDs and Human-Computer Interaction (HCI). The techniques presented in this paper are based on Bowman 
et. al. [7] theory of 3D object manipulation in VEs. Based on the aforementioned theory, we took into account the properties related to the interaction process between user and ETHDs. These factors comprise: input, movement control, displacement and contact as illustrated in Fig. 2.

The design space and its different levels were chosen according to the chronological order of each feature during the interaction process. This design space is divided first by the two input possibilities: motion or controller input. This design space level is justified under the reason that input represents the basic feature of the ETHD interaction process since it determines how the user is going to instruct the system to displace the end-effector to a new position. Movement control is considered on the next level since it involves the task execution. Then, displacement represents the latter part of the task planning since it relates to how the next system's position is going to be reached. On the last level, we considered the contact feature. Normally, ETHDs require to have an intermittent contact with the surface to be touched [29]. In this work we decided to explore the efficiency of this approach but also considered the alternative of being always in contact with end-effector of the device.

A set of five techniques presented in this paper were designed to illustrate different branches of the design space. The diagram presented in Fig. 2 describes the design space organization and the positioning of each interaction technique. In this design space, the branch of automatic action triggered by controller input (right part of the diagram) is discarded since no user input can be used in this case. The properties of the four different levels are described below.

\subsubsection{Input}

This feature represents the input given to the system as a mean to activate the IT or any of its phases. This feature was considered as part of the ITs design space since it represents a fundamental part of the process. The two different branches of this feature are:

- Controller input. The system is controlled with the help of an external device.

- Gesture input. The system is controlled by gestures performed by the users' hands.

\subsubsection{Movement Control}

This feature represents whether the movement is automatic after the command or if it needs manual input to go to the next position for rendering the contact area. The two different branches of this feature are:

- Manual. The user actively chooses the next position and gives the command for the system to move to a determined position.

- Automatic. The system is in charge of automatically displacing the contact area based on the given input.

\subsubsection{Displacement}

This feature represents whether the end-effector can directly be displaced to a selected position or if it can only reach a desired position through gradual movements. The two different branches of this feature are:

- Stepped. The contact area is displaced over gradual steps towards a direction specified by the user.

- Absolute. The user chooses the next position of the contact area: Then, the system displaces physically the end-effector to the previously specified location.

\subsubsection{Contact}

In this feature, the user contact is considered as a variable. It allows to evaluate whether a continuous or intermittent contact could be beneficial for both directing the new area of an ETHD and large surface perception. The two different branches of this feature are:

- In-Contact. The user is in constant contact with the surface while displacing the contact area.

- Out-of-Contact. The user is in intermittent contact with the surface while displacing the contact area.

\subsection{Interaction Techniques}

The interaction techniques presented in this work represent different approaches to current grounded ETHD system capabilities. The techniques address some of the current technological limitations through different approaches. Fig. 3 illustrates the behaviors of the different techniques.

\subsubsection{Swipe}

The Swipe technique takes into account the gesture of swiping the contact area for displacing it. This technique was conceived for providing an interaction similar to the gesture used on touch screens for scrolling through a user interface bigger than the screen. The features of the swiping gesture such as out-of-contact control as well as a fixed and stepped displacement are considered as variables of the design space. The swipe direction is taken into account for displacing the contact area over a predefined distance of $15 \mathrm{~cm}$ in our system (1.5 times the diameter of the contact area). The users can reach the desired position through a series of swipe gestures. This technique was designed for bringing up the familiarity of the gesture on ETHD end-effectors with the gesture on touch screens. The technique profits also from the similar properties of intermittent contact when interacting with touch screens or with ETHD systems.

\subsubsection{Clutch}

The Clutch technique represents the possibility of using a controller as an input method for controlling the system actions. When users can control the ETHD end-effector displacement, they can also acknowledge that the contact area rendering and robot displacement could be managed similarly to many other types of technological devices. The elapsed time and precision of the task fully relies on the user. This technique allows users to initiate the robot's movement in a desired direction to continue their haptic exploration by pressing a controller's trigger. While the trigger is pressed, the user can select the next position of the ETHD system with his hand interacting with the contact area. Releasing the trigger will select and fix the new position. This technique allows the user to know the position of the new contact area and also provides feedback of the elapsed time for the new surface to be loaded. The Clutch technique relies on having an intermittent contact with the surface, thus only allowing contact when the surface is loaded. This technique was conceived considering the clutching procedure for 3D manipulation presented in the work by Bowman et. al [7] and translated to haptic devices in the work of Dominjon et. al. [12]. In our ETHD system, the procedure is mainly divided on pressing and releasing the trigger of the Vive controller. When pressing the controller, users need to raise their finger up and outside the contact area. This gesture activates the projection of the auxiliary contact area, which indicates the next position of the system. Users move their finger to displace this auxiliary area to the next position that they want to touch. Once they make their choice, users release the Vive controller trigger and wait for the area to be rendered. A visual feedback is displayed showing how the first area turns red and disappears while the selected area turns green and appears. After the animation, users can touch the contact area.

\subsubsection{Drag}

The Drag technique consists of displacing the contact area by following the user's finger position. The user triggers the technique by elevating the finger over the surface and selects the desired position by lowering it down. This technique provides feedback of the time taken by the surface to be rendered (see the color modifications in Fig. 3) and also permits the user to know the exact location where the contact area will be next. This technique was inspired from the HCI domain with the drag metaphor that is used for selecting an object and then dragging it into another position [7]. The procedure consists of a series of steps: first, users need to raise their finger up 


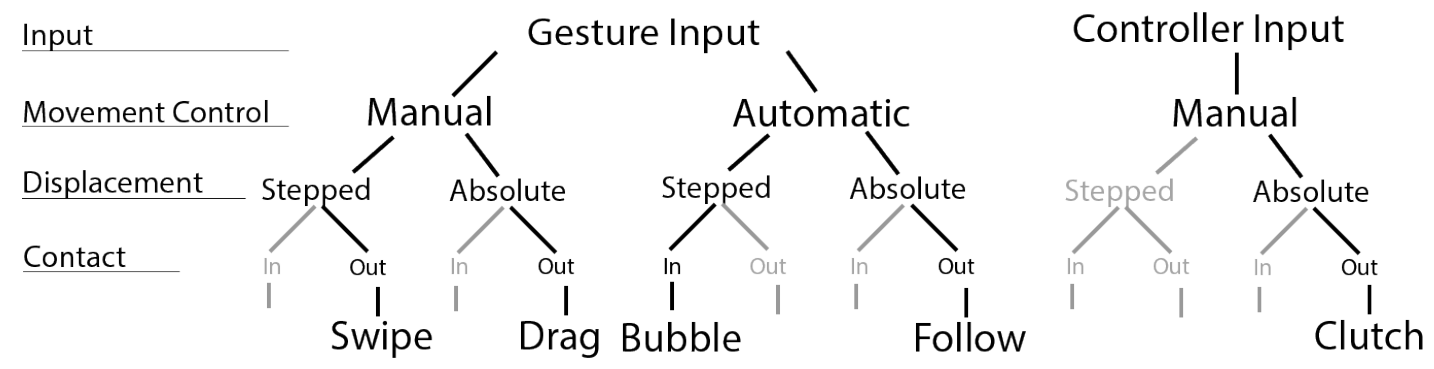

Figure 2: The ETHD Interaction Technique Design Space. This proposed design space is organized with four levels that describe the interactive process between the user and an ETHD system. The first level (input) refers to the input channel mainly used to control the actions of the system. The second level (movement control) describes whether the end-effector's movement is automatic or it needs to be manually triggered. The following level (displacement) refers to whether users can displace the end-effector to a new position using an absolute displacement or they can just move the device through a step series. The last level (contact) describes if users are in constant contact with the end-effector or not.

and outside the contact area. Then, the performed gesture activates the projection of the auxiliary contact area, which indicates the next position of the system. Users then move their finger to displace this auxiliary area to the next position that they want to touch. Once they make their choice, users lower down their finger to activate the area selection. A visual feedback is displayed showing how the first area turns red and disappears while the selected area turns green and appears. After the animation, users can finally touch the contact area. This technique is similar to the Clutch but it differs on the input method. For selecting the next contact area, users have to lower their finger enough to trigger the command of the robot. Then, the robot moves the end-effector below the finger yet high enough for not touching the physical system while this one is moving. Both Drag and Clutch techniques provide visual feedback corresponding to the time taken for the robot to go from one position to another. This feedback helps users to understand the time need to go all over a long distance between two positions.

\subsubsection{Bubble}

The Bubble technique was inspired by the work of Dominjon et. al. [11]. It consists in displacing a "bubble" around the contact area. The contact area is constantly displaced below the user's finger. This technique was designed to take into consideration a constant contact with the end-effector through the surface haptic exploration. To control the motion of the contact area, users need to displace their finger to touch the border of the contact area (or bubble) in order to trigger the movement. The movement is executed in the direction established by the vector starting from the contact area center to the contact point. For deactivating the movement, users need to release their finger and to not touch the border zone. When the users are inside the bubble, they can freely move their fingers without modifying the position of the contact area.

\subsubsection{Follow}

The Follow technique provides constant feedback about the contact area position while it is displacing underneath the user. This technique is close with state-of-the-art methods since the robot tries to reach the user's finger position in real time. The main difference between this technique and state-of-the-art ones is that there is a visual feedback indicating a displacement of the contact area towards the target position. To control the motion of the contact area, users first need to raise their finger up and outside the contact area to activate the technique. While the users' finger is in mid air, the contact area follows the finger's position with respect to the coloring area. The robotic system follows the user through a stepped movement series. The technique is deactivated when the user decides to touch again the virtual contact area.

\subsubsection{Justification}

Different branches of our proposed designed space were explored through the creation of ITs dedicated to address ETHDs' limitations in terms of contact area, lag and unexpected collisions. The limited contact area issue was addressed by relying on the different iterations of our IT design space as well as two types of surface contact: In-contact and out-of-contact. The in-contact branch feature was included to verify if constant contact with a moveable surface renders a sensation of touching a large surface. The out-of-contact feature considers the alternative to be in intermittent contact with the tangible surface as in common ETHD's. The lag issue was addressed by considering two different parameters on the displacement feature in the design space. The stepped displacement branch considers a fast, short movement that happens after the input is received from the user. The absolute displacement feature branch considers a large range movement which indicates the time taken by the robot to arrive from one position to another. Therefore we intended to explore a fast and short movement versus a slower and larger one but with visual progress feedback. The issue of unexpected collisions with users was addressed by visually representing the contact area and adding a color code indicating when the robot was in movement (see Figure 3). Additionally, a visual cue was included to indicate users when an area could not be reached by our system.

\section{Use-CAse}

A use-case was designed for testing the different interaction techniques on a scenario involving the exploration of a wide and flat surface. The considered scenario depicts a desktop surface located in a virtual room (see Fig. 4). The task consists in coloring figures over a canvas. The coloring task involves the exploratory procedures proposed by Lederman \& Klatzky [23] of lateral motion (coloring and being in constant contact with the surface) and pressure (triggering interaction techniques such as Bubble and Swipe). Users can explore the surface with each of the interaction techniques. The surface to be colored displayed three shapes: a circle, a triangle and a square arranged on a canvas. The shape properties, size and their location in the canvas represented different levels of difficulty to carry the task.

Our proposed ETHD is based on a Universal Robots UR5 robotic arm. This robot received constantly trajectory-based linear movement instructions using as parameters the users' fingers position in the VE and the robot's current position. Participants can touch the prop with the tip of their dominant hand's index finger. Participants can use their non-dominant hand for holding the Vive controller required for some interaction techniques.

The system uses a HTC Vive HMD tracker and controller for controlling the 3D interaction. The Vive tracker was attached to a hand strap with and integrated support used to constrain the index 
Swipe:

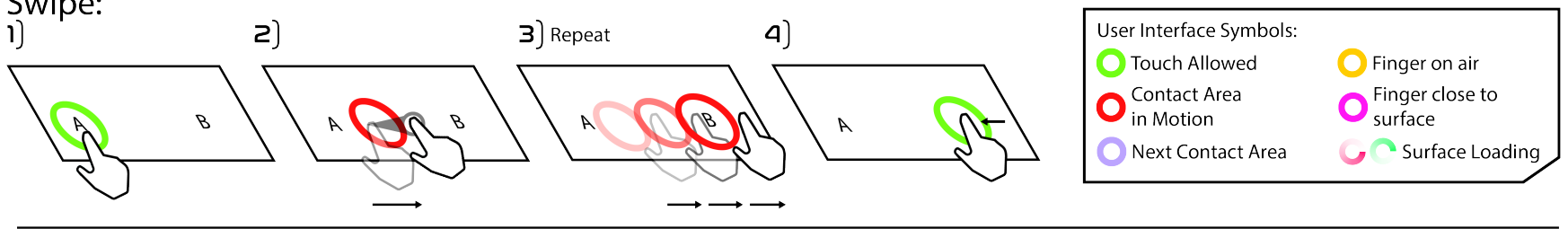

Clutch:

1)

2)

3)

4)

5)

6)

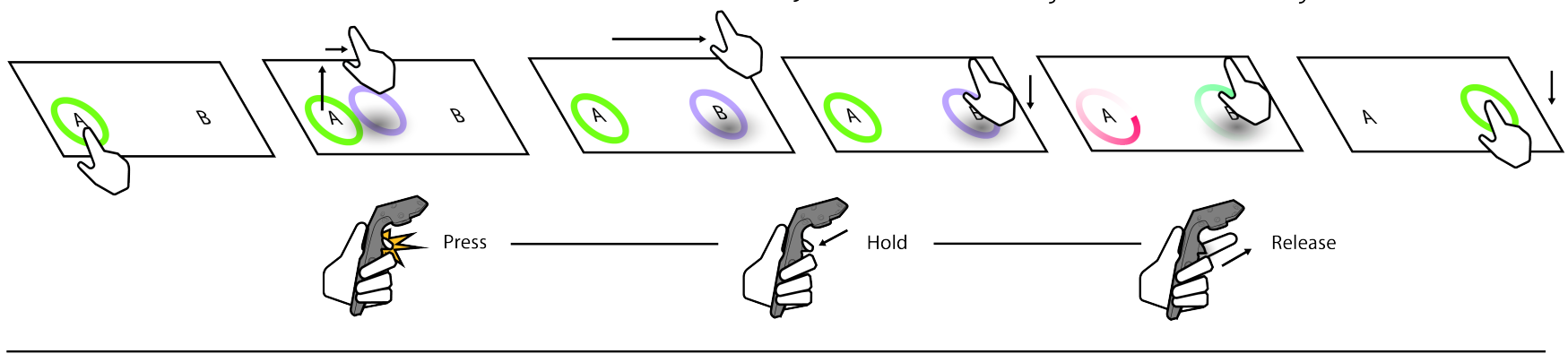

Drag:

1)

2)

3)

4)

5)

6)

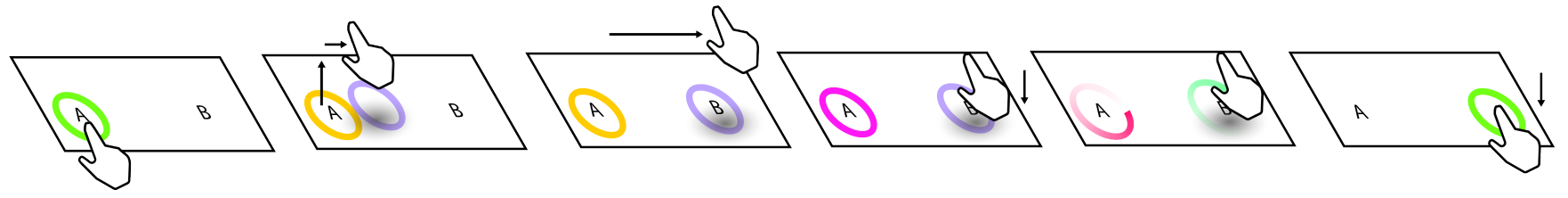

Bubble:

$\begin{array}{llll}\text { 1) 2] 3) } & \text { 3) }\end{array}$

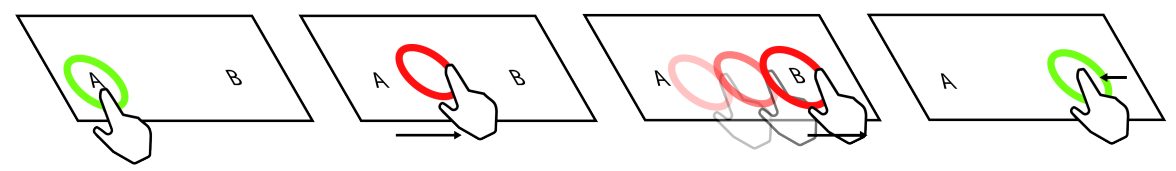

Follow:

1)

2)

3)

4)

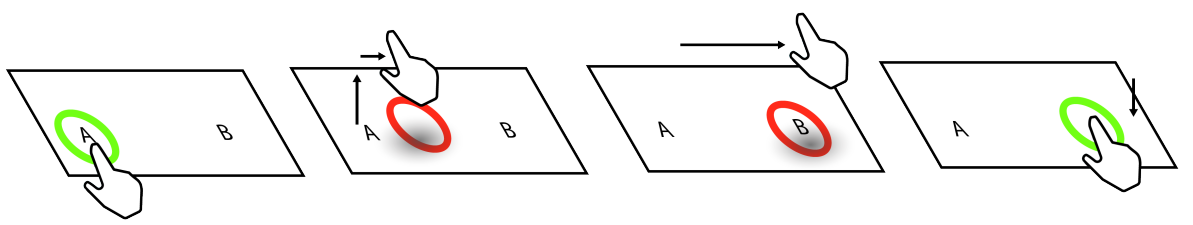

Figure 3: Interaction Techniques. This figure illustrates the different ITs designed by following different branches of our design space. The Swipe technique consists on performing a swipe gesture for displacing the contact area. The Clutch technique uses the Vive controller to trigger the contact area selection and displacement. The Drag technique permits the user to select and displace the area to be touched. The Bubble technique relies on touching the border of the contact area to displace it over a fixed distance. The Follow technique automatically follows the user finger while the latter is hovering on air. For more details please refer to the techniques description in subsection 3.2. 


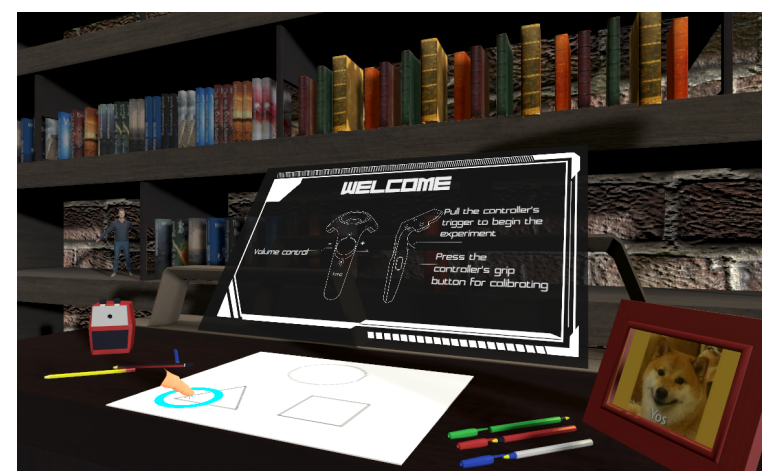

Figure 4: The VE depicting the use-case scenario. The environment was designed to give context to task of coloring/painting.

finger movement to favor the virtual representation of the finger and its positioning in the VE. The environment was created using Unity. Robot's communication with Unity is achieved through TCP port communication and parsing commands to the UR5 programming language and interface. The virtual simulation was run on a computer with an Intel Core i7-7820HQ processor with an NVIDIA Quadro P3000 graphics processor.

\section{USER STUdY}

A user study was conducted to assess the performance and user experience for each interaction technique in the task of shape coloring in a virtual surface. Participants responses were used to have a deeper insight of users perception for each technique in terms of precision, usability and efficiency.

\subsection{Experimental Setup}

We designed an experimental prop which consists on a round flat surface attached to the UR5 end-effector (see Fig. 1). The 3D printed surface has a radius of $8.25 \mathrm{~cm}$. This radius size was motivated by adding an additional space to be touched outside the virtual contact area which radius was $5 \mathrm{~cm}$. This extra space was also considered for not disclosing the limited size of the prop's surface while performing the gestures for each interaction technique. The experimental setup is shown in Fig. 1. Both prop and experimental setup were used for the use-case scenario previously described in Section 4.

\subsection{Procedure}

Participants were instructed about how to use each interaction technique without disclosing its concept name. Once participants confirmed that they understood each technique, they proceeded to begin the experiment. For each condition, participants were required to complete a training task in order to get familiarized with each technique. Participants were asked to wear an isolating headphone set displaying white noise when participating in the experiment as a mean to not perceive sounds produced by the robot while displacing the end-effector. They could also control the volume in a range where they could not hear the robot noise without being annoyed nor distracted by the white noise. The participants were asked to paint the three shapes using their index finger. They were allowed to displace the contact surface by using the tested interaction technique. Their objective was to be as accurate as possible during their painting task.

\subsection{Experimental Design}

We considered the five ITs presented in Section 3. Each of these techniques were used as conditions for the virtual painting task. The order of these conditions was sorted using the Latin Squares method.

The experiment consisted of 5 blocks with 1 training trial and 3 experimental trials. Giving a total of 20 trials. Each trial had a maximum duration of 4 minutes. Participants were aware of the elapsed time during each trial by watching an integrated chronometer in the main UI. The 5 blocks were presented using the Latin Squares method.

\subsection{Collected Data and Population}

The collected data for this study concerned both results of the painting task performance and user experience (UX). The elapsed time for each trial was registered along with the paintings generated for each trial. The paintings were analyzed to count the quantity of painting inside $\left(S_{i}\right)$ for every shape $(j)$ (See Equation 1). Additionally, the overall quantity of spilled painting outside the shapes' borders $\left(S_{e}\right)$. These factors were considered to calculate an error ratio (See Equation 2).

$$
S_{i}=\sum_{j}^{1} S_{i j} \quad(1) \quad \text { and } \quad \frac{S_{e}}{S_{i}+S_{e}}
$$

Thereafter, we will use this ratio and express it as a percentage (named Painting Error Percentage). After the experiment, participants were asked to fill a user experience questionnaire (UEQ). This questionnaire integrated questions from [17] and [30], focusing on usability factors by asking the participants whether they considered the ITs as controllable, captivating, innovative, pleasant, motivating, easy to learn, and fast. The answer format for these questions was presented on a Likert scale. We gathered a total of 20 participants (6 female, 14 male), ages 22 to $33(\mathrm{M}=26.45, \mathrm{SD}=3.236)$.

\subsection{Results}

The paintings produced by each participant were analyzed to assess the total amount of surface covered for all the shapes and the total amount of spilled paint outside the borders (See Fig. 5 for an illustration of three paintings with different levels of performance). The performance is summarized in Table 1 .

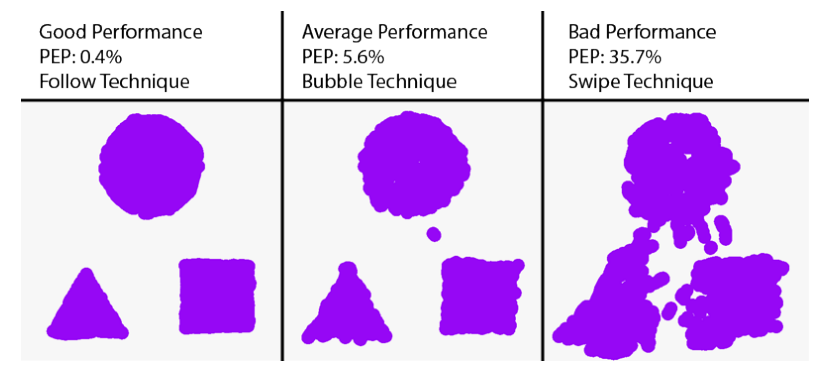

Figure 5: The resulting participants' paintings. The paintings were analyzed to count the number of pixels colored inside the shapes as well the outside ones to determine the performance for each technique. A good performance painting is shown on the left. An average performance is shown the middle while a bad performance is shown on the right.

To study the time needed by the participants to perform the task, we used a linear mixed model on the collected data with respect to the ITs. The participants were considered as a random effect in the model. Our analysis of variance showed a significant effect on the ITs $(F(4,262)=6.37, p<0.001)$. We performed a posthoc analysis on the ITs using Tukey tests. Bonferroni correction was used for all post-hoc analysis. The post-hoc analysis revealed significant differences between the Swipe technique and: the Bubble technique $(p<0.001)$, the Clutch technique $(p<0.001)$ and the Follow technique $(p=0.02)$. We found also significant differences between the Drag technique and both Clutch $(p=0.005)$ and Bubble techniques $(p=0.009)$. 


\begin{tabular}{lllll}
\hline IT & Time & PEP & ESCP & ISCP \\
\hline Swipe & $126(38.4)$ & $7.4 \%(4.8 \%)$ & $2.1 \%(1.6 \%)$ & $99.1 \%(1.1 \%)$ \\
Clutch & $102(30.4)$ & $4.5 \%(4 \%)$ & $1.2 \%(1.2 \%)$ & $99.4 \%(0.7 \%)$ \\
Drag & $118(39.1)$ & $4.7 \%(3.7 \%)$ & $1.3 \%(1 \%)$ & $99 \%(1.2 \%)$ \\
Bubble & $103(33.2)$ & $6.8 \%(4.2 \%)$ & $1.9 \%(1.3 \%)$ & $99.3(0.8 \%)$ \\
Follow & $113(39.5)$ & $4.9 \%(3.6 \%)$ & $1.3 \%(1.1 \%)$ & $99.4(0.7 \%)$ \\
\hline
\end{tabular}

Table 1: Performance results for elapsed time and painting quality. The results (mean and standard deviation) are: average time (in seconds), painting error percentage (PEP), exterior shape coloring percentage (ESCP) and interior shape coloring percentage (ISCP). The PEP refers to a ratio considering both ESCP and ISCP defined previously in Equation 2.

Concerning the analysis of the PEP, we used a linear mixed model on the collected data with respect to the ITs. The participants were considered as a random effect in the model. Our analysis of variance showed a significant effect on the ITs $(F(4,262)=16.51$, $p<0.001)$. The post-hoc analysis using Tukey tests revealed significant differences between the Swipe technique and: the Drag technique $(p<0.001)$, the Clutch technique $(p<0.001)$ and the Follow technique $(p<0.001)$. We also found significant differences between the Bubble technique and: the Swipe technique and: the Drag technique $(p<0.001)$, the Clutch technique $(p<0.001)$ and the Follow technique $(p<0.001)$. Results on the subjective questionnaire are summarized in Fig. 6.

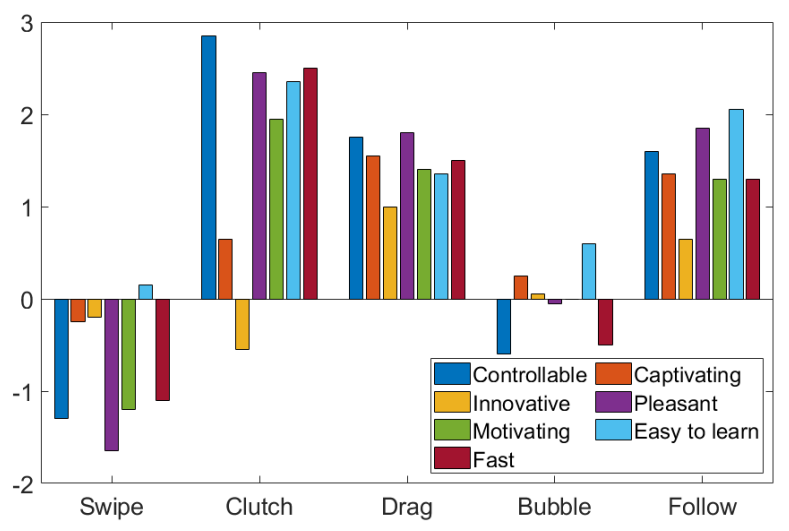

Figure 6: User experience questionnaire results. The average scores for several features evaluated in the subjective questionnaire. The results are depicted on a scale ranging from -3 to 3 .

To study the answers to the subjective questionnaire, we used linear mixed models on the collected data for each of the 7 criteria, with respect to the ITs. The participants were considered as a random effect in the model. Our analysis of variance showed a significant effect on the ITs for 5 criteria: Controllable $(F(4,76)=7.62$, $p<0.001)$, Captivating $(F(4,95)=15.28, p<0.001)$, Motivating $(F(4,76)=5.96, p<0.001)$, Easy to learn $(F(4,76)=6.043$, $p<0.001)$, and Fast $(F(4,76)=5.96, p<0.001)$. No significant effect was found for the Innovative criterion $(p=0.17)$ and the Pleasant one $(p=0.32)$. However, several participants qualified the Drag as an innovative technique. In their own words: "Favourite technique due to the time guidance for the activation of the zone", "The one I loved the most! Most innovative one and very quick with good control", "Great one, pretty convenient and one-handed".

Concerning the Controllable criterion, post-hoc analysis revealed a significant difference between the Clutch technique and: the Bubble technique $(p<0.001)$, the Follow technique $(p=0.006)$ and the Swipe technique $(p=0.003)$. There was also a significant effect between the Drag technique and: the Bubble technique $(<0.001)$, the
Follow technique ( $p=0.004)$ and the Swipe technique $(p=0.002)$. Participants qualified the Clutch technique as the most controllable, yet the most conventional. In their own words: "Easy to control, but need an extra hand", "Nice, simple, the trigger gives you control, you decide WHEN you want to move the circle", "Intuitive but perhaps "old-fashioned" in this type of VR environment".

Concerning the Captivating criterion, post-hoc analysis revealed a significant difference between the Bubble technique and: the Drag technique $(p<0.001)$, the Clutch technique $(p=0.002)$ and the Follow technique $(p=0.03)$. A significant effect was also found between the Swipe technique and: the Drag technique $(p<0.001)$ and the Clutch technique $(p=0.03)$. Finally, a significant effect was found between the Drag technique and: the Follow technique $(p<0.001)$ and the Clutch technique $(p<0.001)$. Participants rated both Drag and Follow as the most captivating techniques.

Concerning the Motivating criterion, post-hoc analysis revealed a significant difference between the Swipe technique and all other ITs: Bubble $(p<0.002)$, Clutch $(p<0.001)$, Drag $(p=0.007)$ and Follow $(p<0.001)$. The Swipe technique received comments from the users concerning their frustration of not being able to control or to extend the distance with a stronger gesture. In their own words "I really don't like it. It was frustrating not being able to control how large is the swipe movement".

Concerning the Easy to learn criterion, post-hoc analysis revealed a significant difference between the Drag Technique and: the Bubble technique $(p<0.001)$, the Clutch technique $(p=0.03)$ and the Follow technique $(p<0.001)$. There was also a significant difference between the Swipe technique and: the Bubble technique $(p=0.002)$ and the Follow technique ( $p=0.01)$. According to participants' comments, the Clutch technique was noted as the easiest technique to learn. In their own words: "Fast progression with this one, perhaps for people with VR experience", "Using a controller for changing painting mode to moving mode was as easy as the method C (Drag)". Concerning the Swipe technique, participants commented that its concept was easy to understand but that it took them some time to master it.

Concerning the Fast criterion, post-hoc analysis revealed a significant difference between the Bubble technique and: the Drag technique $(p=0.01)$, the Follow technique $(p=0.01)$ and the Swipe technique $(p<0.001)$. There was also a significant difference between the Swipe technique and the other techniques: Clutch $(p<0.001)$, Drag $(p=0.03)$ and Follow $(p=0.03)$. Both Bubble and Swipe techniques implemented the stepped displacement. Participants commented that they appreciated the techniques format, but they made emphasis on the time that it took them to reach a desired position. In their own words: "(Bubble) Good control, but a little bit slow". In the case of the Bubble technique, these comments contrast with the fact that this technique achieved the second shortest average trial time.

Concerning other factors such as presence in the VE, we informally received feedback from the users arguing that the Clutch and Drag techniques were the ones providing a more realistic experience to touching a flat, wide surface. In their own words: "I think it (Drag) offers the best compromise in terms of immersion and performance, since it feels very precise". And "(Clutch) Using the controller blocks one hand and kind of breaks immersion".

\section{Discussion}

Our paper studies a design space where several ITs can be conceived by combining diverse parameters aiming to address the common issues that ETHDs present, namely, lag, limited, contact area and unexpected collisions. For instance, the displacement feature in the design space addresses lag and contact area limited size by considering providing a stepped but immediate end-effector displacement and thus rendering in a fast manner a new area to touch. The integrated visual feedback indicates the system's current position to 
avoid unexpected collisions and to inform users about the size and location of the contact area.

The techniques studied in this paper are an exploration of several branches of the design space. The possible combinations provided by the design space are too many to be properly evaluated in one study. Thus, we decided to implement techniques that could represent at least one the features proposed in the design space. Future work could explore new techniques conceived under our design space to study their performance in different scenarios and tasks. Under this framework, we already designed a set of 5 techniques: Swipe, Clutch, Drag, Bubble, Follow. To further study the performance of these techniques, a use-case scenario was designed to contextualize a task that required rendering a large contact area and a constant interaction between user and surface. The chosen task was to color shapes with the index finger since it is adapted to the system's configuration and to an interaction that requires large surface exploration and constant contact with the surface. A user study was conducted with the purpose of assessing the coloring task performance and user experience. This was done as mean to understand the efficiency and usability of our techniques on a context adapted to ETHDs as well as a mean to test different possibilities of our design space.

Primarily due to safety reasons, users need to be aware that they are interacting with a grounded robotic system. Therefore, we proposed ITs with a dedicated visual feedback. Providing feedback that indicates when to touch the surface and the process of translating the end-effector to another location is useful for users to cope with the system limitations. Thus, comments from users pointed out that visual feedback was appreciated as it helped them to understand when they could be able to touch the contact area. The feedback corresponding to contact area loading has already been considered in the work of Abtahi et. al. [3] and Posselt et. al [29]. The feedback corresponding to location selection that involves absolute end-effector displacement such as in Clutch, Drag and Follow techniques has also been considered in the work of [29] where users were able to select the texture to be touched so the robot could afterwards move to a position where the texture could be located. Results from the user study suggest that absolute end-effector displacement performs better than an immediate short stepped displacement. These techniques additionally had the lowest scores concerning painting error.

The integration of gesture commands to displace the contact area of an ETHD has been considered in previous work [6, 15]. Incorporating gesture-based interaction techniques requires considering the differences between the gestures and the hand-movements that will occur during the interaction. Results from this study suggest higher performance when gestures are mostly different from coloring the task such as the ones used on Drag or Clutch techniques. On the opposite side, the similarity between the swipe and coloring gestures confused users at the moment of learning how to control the interaction technique on the experimental task. The integration of a controlling device for ETHD interaction could be taken into account when training the user to learn to control the system. The inclusion of the controller does not have to interfere with the haptic perception but only to work as a tool for selection. Comments from users suggested that the controller helped them to separate the task of selecting the contact area and touching the surface. Users qualified Drag as the most innovative technique since it does not involve a controller and overall it was the second best rated technique. Remarks from participants over Drag suggested the opportunity of changing the gesture of lowering down the finger for selecting the contact area to a more precise gesture. Results from this study suggest that the Drag technique (which shares almost every feature with Clutch except the usage of a controller) can be considered as a technique to be integrated in the future. The arguments in favor of this technique mostly rely on the usage of natural gestures to point and select the contact area. Results in time performance indicate the Drag and Follow techniques had the greater amount of time elapsed by the users. This might be due that this techniques required pointing towards the desired contact area [28,32] and Fitts' Law [7, 14, 16, 25] as shown in the research of Bruder et al. [9] and Chun et al [22]. In addition, the presented shapes had also different levels of complexity in the coloring task. For instance, the triangle had narrow angles that complicated the task but the initial position was always close to this shape. The circle on the other hand was the easiest to color since there were no corners and it coincided with the brush shape yet it was the furthest shape from the starting point. The square provided a balance between difficulty, size and distance to the user. The work of Arora et al. [5] suggests to take into consideration the complexity of the shape to be colored in the task. Efforts focused on 2D interaction for ETHDs also looked for increasing the contact area through active surface rendering such as physical end-effector rotation $[27,34]$ and pin-array based surface rendering [15]. Other methods have been considered such as user virtual redirection [33]. While the Drag and Clutch techniques had a good performance in the user study, we recognize that their features can be challenged in other scenarios. The proposed set of techniques could be further studied concerning their performance in conditions where the geometry to be explored possess more complex properties. For instance, the Bubble technique could not be suited for curved surface exploration. Through the use of our design space, future work could consist in designing new ITs and testing them in other scenarios. Combining ETHDs and VEs can lead to optimize the amount of tangible surface needed to provide a convincing sensation of touch in a virtual simulation. Results from this study suggest that an adequate IT that profits from the ETHD features can not only provide the sensation of touching a large object but it also optimize task performance and UX. Besides task performance and UX, others factors are worth to be explored in future work. Namely, overall ETHD efficiency in providing haptic feedback in VR, ETHDs performance against other types of haptic displays such as wearable ones, and user presence in a system combining both ETHDs and VEs.

\section{Conclusion}

ETHDs can render haptic feedback without constraining users to wear an actuator. Nevertheless, ETHD systems need to overcome issues concerning primarily large contact area rendering, lag and unexpected collisions with users. To address these issues, we proposed in this paper a design space considering features that can be explored to conceive ITs for ETHDs. These features are: input, movement control, displacement and contact. Using our design space, we explored several feature combinations conceiving a set of 5 techniques: Swipe, Clutch, Drag, Bubble and Follow.

A use-case scenario was created in order to contextualize the application of these techniques. The task of surface coloring over a digital canvas was chosen as it involves a large and constant surface exploration. To assess the techniques performance, a user-study was conducted to evaluate the accuracy and elapsed time taken for a shape coloring task. In addition, we qualified the UX perceived by users as a mean to identify the features that were better evaluated by users. Results point out that the Clutch and Drag techniques had a good performance in matters of low PEP and UX. The features shared by these techniques are the absolute displacement, out-of-contact triggering and user-controlled motion. Participants commented that the visual feedback displayed with these techniques helped them to better understand the surface rendering process.

Taken together, our results and design space framework could help to the design of novel ITs adapted to a wider range of possible tasks. The features considered for the design space can be increased or adapted to enrich the UX with the purpose of coping with the devices' limitations. Future work will be focused on designing and evaluating ITs for more complex volume exploration as well as various scenarios. 


\section{ACKNOWLEDGMENTS}

The authors would like to thank the French National Research Agency (ANR) for funding the LobbyBot project.

\section{REFERENCES}

[1] M. Abdullah, M. Kim, W. Hassan, Y. Kuroda, and S. Jeon. HapticDrone: An Encountered-Type Kinesthetic Haptic Interface with Controllable Force Feedback: Initial Example for 1d Haptic Feedback. In Proc. UIST, pp. 115-117. ACM, 2017. doi: 10.1145/3131785.3131821

[2] P. Abtahi and S. Follmer. Visuo-Haptic Illusions for Improving the Perceived Performance of Shape Displays. In Proc. CHI, pp. 1-13. ACM, 2018. doi: 10.1145/3173574.3173724

[3] P. Abtahi, B. Landry, J. J. Yang, M. Pavone, S. Follmer, and J. A. Landay. Beyond The Force: Using Quadcopters to Appropriate Objects and the Environment for Haptics in Virtual Reality. In Proc. CHI, pp. 1-13, 2019. doi: 10.1145/3290605.3300589

[4] B. Araujo, R. Jota, V. Perumal, J. X. Yao, K. Singh, and D. Wigdor. Snake Charmer: Physically Enabling Virtual Objects. In Proc. TEI, pp. 218-226. ACM, 2016. doi: 10.1145/2839462.2839484

[5] R. Arora, R. H. Kazi, F. Anderson, T. Grossman, K. Singh, and G. Fitzmaurice. Experimental Evaluation of Sketching on Surfaces in VR. In Proc. of CHI, pp. 5643-5654, 2017. doi: 10.1145/3025453.3025474

[6] A. Boem and H. Iwata. Encounter-Type Haptic Interfaces for Virtual Reality Musical Instruments. In Proc. 3DUI, pp. 1-2. IEEE, Mar. 2018 doi: 10.1109/VR.2018.8446549

[7] D. A. Bowman, E. Kruijff, J. J. LaViola, and I. Poupyrev. AddisonWesley, Boston, MA, 2005. OCLC: 879357696.

[8] D. Brice, S. Devine, and K. Rafferty. A Novel Force Feedback Haptics System with Application in Phobia Treatment. In Proc. WSCG. ACM, 2017.

[9] G. Bruder, F. Steinicke, and W. Sturzlinger. Effects of visual conflicts on 3D selection task performance in stereoscopic display environments. In Proc. of 3DUI, pp. 115-118. IEEE, 2013. doi: 10.1109/3DUI.2013. 6550207

[10] S. Devine, K. Rafferty, and D. Ferguson. "HapticVive" - A Point Contact Encounter Haptic Solution with the HTC VIVE and Baxter Robot. In Proc. WSCG. ACM, 2017.

[11] L. Dominjon, A. Lecuyer, J. Burkhardt, G. Andrade-Barroso, and S. Richir. The "Bubble" Technique: Interacting with Large Virtual Environments Using Haptic Devices with Limited Workspace. In Proc. Eurohaptics, pp. 639-640. IEEE, 2005. doi: 10.1109/WHC.2005.126

[12] L. Dominjon, A. Lécuyer, J.-M. Burkhardt, and S. Richir. A comparison of three techniques to interact in large virtual environments using haptic devices with limited workspace. In T. Nishita, Q. Peng, and H.-P. Seidel, eds., Advances in Computer Graphics, pp. 288-299. Springer, 2006.

[13] A. Filippeschi, F. Brizzi, E. Ruffaldi, J. M. Jacinto, and C. A. Avizzano Encountered-type haptic interface for virtual interaction with real objects based on implicit surface haptic rendering for remote palpation. In Proc. of IROS, pp. 5904-5909. IEEE/RSJ, 2015. doi: 10.1109/IROS .2015 .7354216

[14] P. M. Fitts. The information capacity of the human motor system in controlling the amplitude of movement. Journal of Experimental Psychology, 47(6):381-391, 1954. doi: 10.1037/h0055392

[15] D. Fitzgerald and H. Ishii. Mediate: A Spatial Tangible Interface for Mixed Reality. In Proc. CHI, pp. 1-6. ACM, 2018. doi: 10.1145/ 3170427.3188472

[16] M. J. Fu, A. D. Hershberger, K. Sano, and M. C. Cavusoglu. Effect of visuo-haptic co-location on 3D Fitts' task performance. In Proc. of IROS, pp. 3460-3467. IEEE, 2011. doi: 10.1109/IROS.2011.6094707

[17] M. Hassenzahl. The Interplay of Beauty, Goodness, and Usability in Interactive Products. Human-Computer Interaction, 19(4):319-349, 2004. doi: 10.1207/s15327051hci1904_2

[18] M. Hoppe, P. Knierim, T. Kosch, M. Funk, L. Futami, S. Schneegass, N. Henze, A. Schmidt, and T. Machulla. VRHapticDrones: Providing Haptics in Virtual Reality through Quadcopters. In Proc. MUM. ACM, 2018. doi: 10.1145/3282894.3282898

[19] Y. Kim, H. J. Kim, and Y. J. Kim. Encountered-type haptic display for large VR environment using per-plane reachability maps: Encountered- type Haptic Display for Large VR Environment. Computer Animation and Virtual Worlds, 29(3-4), 2018. doi: 10.1002/cav.1814

[20] P. Knierim, T. Kosch, A. Achberger, and M. Funk. Flyables: Exploring 3d Interaction Spaces for Levitating Tangibles. In Proc. TEI, pp. 329336, 2018. doi: 10.1145/3173225.3173273

[21] P. Knierim, T. Kosch, V. Schwind, M. Funk, F. Kiss, S. Schneegass, and N. Henze. Tactile Drones - Providing Immersive Tactile Feedback in Virtual Reality through Quadcopters. In Proc. CHI, pp. 433-436, 2017. doi: $10.1145 / 3027063.3050426$

[22] Kwonsoo Chun, B. Verplank, F. Barbagli, and K. Salisbury. Evaluating haptics and 3D stereo displays using Fitts' law. In Proc. of C5, pp. 53-58. IEEE, 2004. doi: 10.1109/HAVE.2004.1391881

[23] S. J. Lederman and R. L. Klatzky. Hand movements: A window into haptic object recognition. Cognitive Psychology, 19(3):342-368, July 1987. doi: 10.1016/0010-0285(87)90008-9

[24] C.-G. Lee, G. L. Dunn, I. Oakley, and J. Ryu. Visual Guidance for Encountered Type Haptic Display: A feasibility study. In Proc. ISMAR, pp. 74-77. IEEE, Sept. 2016. doi: 10.1109/ISMAR-Adjunct.2016. 0044

[25] C. L. MacKenzie, R. G. Marteniuk, C. Dugas, D. Liske, and B. Eickmeier. Three-Dimensional Movement Trajectories in Fitts' Task: Implications for Control. The Quarterly Journal of Experimental Psychology Section A, 39(4):629-647, 1987. doi: 10.1080/14640748708401806

[26] W. McNeely. Robotic graphics: a new approach to force feedback for virtual reality. In Proc of VRAIS, pp. 336-341. IEEE, 1993. doi: 10. 1109/VRAIS. 1993.380761

[27] V. R. Mercado, M. Marchal, and A. Lecuyer. ENTROPiA: Towards Infinite Surface Haptic Displays in Virtual Reality Using EncounteredType Rotating Props. IEEE Transactions on Visualization and Computer Graphics, pp. 1-1, 2020. doi: 10.1109/TVCG.2019.2963190

[28] A. Murata and H. Iwase. Extending Fitts' law to a three-dimensional pointing task. Human Movement Science, 20(6):791-805, Dec. 2001. doi: 10.1016/S0167-9457(01)00058-6

[29] J. Posselt, L. Dominjon, A. Bouchet, and A. Kemeny. Toward virtual touch: investigating encounter -type haptics for perceived quality assessment in the automotive industry. In Proc. EuroVR, 2017.

[30] M. Schrepp, A. Hinderks, and J. Thomaschewski. Applying the User Experience Questionnaire (UEQ) in Different Evaluation Scenarios. In D. Hutchison, T. Kanade, J. Kittler, J. M. Kleinberg, A. Kobsa, F. Mattern, J. C. Mitchell, M. Naor, O. Nierstrasz, C. Pandu Rangan, B. Steffen, D. Terzopoulos, D. Tygar, G. Weikum, and A. Marcus, eds., Design, User Experience, and Usability. Theories, Methods, and Tools for Designing the User Experience, vol. 8517, pp. 383-392. Springer, 2014. doi: 10.1007/978-3-319-07668-3_37

[31] S. Tachi, T. Maeda, R. Hirata, and H. Hoshino. A Construction Method of Virtual Haptics Space. In Proc. ICAT, pp. 131-138. Springer, 1994.

[32] R. J. Teather and W. Stuerzlinger. Pointing at 3D targets in a stereo head-tracked virtual environment. In Proc. of 3DUI, pp. 87-94. IEEE, 2011. doi: 10.1109/3DUI.2011.5759222

[33] E. Vonach, C. Gatterer, and H. Kaufmann. VRRobot: Robot actuated props in an infinite virtual environment. In Proc. VR, pp. 74-83. IEEE, 2017. doi: 10.1109/VR.2017.7892233

[34] E. Whitmire, H. Benko, C. Holz, E. Ofek, and M. Sinclair. Haptic Revolver: Touch, Shear, Texture, and Shape Rendering on a VR Controller. In Proc. CHI, pp. 1-4. ACM, 2018. doi: 10.1145/3170427. 3186515

[35] S. Yamaguchi, H. Shionoiri, T. Nakamura, and H. Kajimoto. An Encounter Type VR System Aimed at Exhibiting Wall Material Samples for Show House. In Proc. ISS, pp. 321-326. ACM, 2018. doi: 10. 1145/3279778.3279908

[36] Y. Yokokohji, R. Hollis, and T. Kanade. What you can see is what you can feel-development of a visual/haptic interface to virtual environment. In Proc. VR, pp. 46-53. IEEE, 1996. doi: 10.1109/VRAIS. 1996.490509 